\title{
Rapid Vaccine Distribution in Nontraditional Settings: Lessons Learned From Project VIVA
}

\author{
Micaela H. Coady, MS \\ Center for Urban Epidemiologic Studies \\ New York Academy of Medicine \\ Linda Weiss, $\mathrm{PhD}$ \\ Division of Health Policy \\ New York Academy of Medicine \\ Sandro Galea, MD, DrPH \\ Center for Urban Epidemiologic Studies \\ New York Academy of Medicine and \\ Department of Epidemiology \\ University of Michigan School of Public Health
Danielle C. Ompad, PhD and Kathryn Glidden, RN
Center for Urban Epidemiologic Studies
New York Academy of Medicine
David Vlahov, $\mathrm{PhD}$
Center for Urban Epidemiologic Studies
New York Academy of Medicine \\ Project VIVA Intervention Working Group
}

With growing fear of a worldwide influenza pandemic, programs that can rapidly vaccinate a broad range of persons are urgently needed. Vaccination rates are low among disadvantaged and hard-to-reach populations living within urban communities, and delivering vaccines to these groups may prove challenging. Project VIVA ${ }^{1}$ (Venue-Intensive Vaccination for Adults), staffed by teams of nurses and outreach workers, aimed to deliver vaccines rapidly within disadvantaged neighborhoods in New York City. Project VIVA nurses offered

Correspondence should be sent to Micaela H. Coady, Center for Urban Epidemiologic Studies, New York Academy of Medicine, 1216 Fifth Avenue, New York, NY 10029. E-mail: mcoady@nyam.org

${ }^{1}$ This project was developed by members of the Harlem Community and Academic Partnership (HCAP) and the Center for Urban Epidemiologic Studies (CUES). The VIVA Intervention Working Group members are: Dr. Ann Boyer, Dr. Robert Brackbill, Brian Brown, Jose Caraballo, Karyn London, Gail Love, Pat Monahan, Dr. Erica Phillips, Sarah Sisco, and Dr. Sharon Stancliff. This work was supported by a grant from the National Institute on Drub Abuse (DA017004) and the Merck Foundation. 
free influenza vaccine door-to-door and on street corners over 10 days in October, 2005. A total of 1,648 people were vaccinated, exceeding expectation. Careful selection and training of project staff, community involvement in project development, community outreach, and prioritizing street-based distribution may be key factors in an effective rapid vaccination program. In conclusion, this project may be replicated in other communities and utilized for annual vaccination campaigns and in the event of a pandemic.

With increasing concerns about the threat of a human influenza pandemic, there is mounting pressure for public health officials to identify new and effective methods to rapidly deliver vaccines to large numbers of people (Cinti, 2005). Community health nurses are likely to play a vital role during a pandemic, including involvement in mass vaccination efforts (Ho \& Parker, 2006). Although there have been a number of replicable vaccination interventions (D’Heilly, Lockman, \& Nichol, 2004; Osterholm, 2001; Poland, Grabenstein, \& Neff, 2005), examples of programs providing rapid vaccinations to hard-to-reach populations (e.g., substance users, elderly shut-ins, homeless) are limited (Weatherill, Buxton, \& Daly, 2004). The recent failure of the smallpox vaccination program among US health care workers, a population that should be receptive to vaccination and highly accessible, highlights the challenges in conducting mass vaccination campaigns (Kuhles \& Ackman, 2003).

A variety of approaches have been employed to improve vaccination coverage, including provider education (Findley et al., 2003); patient education, incentives, and reminders (Hoekstra et al., 1998; Krieger, Castorina, Walls, Weaver, \& Ciske, 2000); and provision of vaccines in settings serving high risk populations, including hospital emergency departments (Slobodkin, Kitlas, \& Zielske, 1998) and syringe exchange sites (Des Jarlais et al., 2001; Stancliff, Salomon, Perlman, \& Russell, 2000). Low vaccination coverage among minorities and other hard-to-reach populations remains a persistent problem, however. Unvaccinated groups within a larger population may propagate disease, particularly in the event of a pandemic (Centers for Disease Control and Prevention, 2003; Egede \& Zheng, 2003; Kuo, Sherman, Thomas, \& Strathdee, 2004).

Building on the lessons learned from the previously-described efforts, Project VIVA was a community-based demonstration project designed to evaluate the feasibility of a rapid vaccination protocol for hard-to-reach populations that could serve as a model for pandemic preparedness plans. For the purposes of this project, hard-to-reach populations included substance users, elderly, undocumented immigrants, the homeless, and commercial sex workers. This community-based intervention, in which community nurses offered door-to-door and street-based vaccinations, successfully delivered 1,648 influenza vaccines in low-income communities of New York City in October, 2005. Here, we describe the project, highlighting the central role played by community-based nurses, to demonstrate its feasibility and outline lessons learned that may guide project replication in other venues. 


\section{PROGRAM DESCRIPTION}

\section{Start-Up}

The demonstration project described here was developed and implemented by a research-community partnership, the Harlem Community and Academic Partnership, which includes researchers at the Center for Urban Epidemiologic Studies, The New York Academy of Medicine (NYAM). The overall goals of Project VIVA were (a) assessment of personal and structural barriers to immunization; (b) examination of the feasibility, acceptance, and utility of a community-public health partnership as a framework for rapid vaccination of hard-to-reach populations; (c) development, implementation, and assessment of a rapid vaccination protocol for hard-to-reach and high-risk populations that could contribute to pandemic influenza preparedness plans; and (d) generalizing this effort to other hard-to-reach populations in other urban areas.

During the first year of implementation, VIVA staff conducted extensive outreach to community members, organizations, and leaders. Outreach workers handed out over 28,000 informational flyers on street corners and delivered 19 presentations at community-based organizations. Staff members also conducted outreach to local community leaders working in housing, HIV/AIDS, needle exchange, and other service delivery programs. Community leaders were invited to participate in relevant project meetings and asked to disseminate project information.

During the first year of the project, those involved also developed methods for enumerating hard-to-reach populations in target neighborhoods and carried out surveys to examine key personal and structural barriers to local vaccination. The survey instrument assessed (a) sociodemographic characteristics, including age, gender, race, and education domains; (b) characteristics that may be associated with social marginalization, including substance abuse and commercial sex work; (c) access to and utilization of health care; (d) vaccination history; (e) general health; and (f) trust in government and social agencies. The anonymous, cross-sectional survey lasted approximately $5 \mathrm{~min}$. and was administered on the street or in apartment buildings in both English and Spanish.

As reported in a prior publication (Bryant et al., 2006), people involved in the project found that those who did not regularly utilize health or social services and those without government-sponsored health insurance were less likely to have been vaccinated. If vaccine was available, however, these individuals would be willing to receive it.

Nurses provided vaccination with influenza vaccine to a limited number of individuals in the start-up phase, to assess likely acceptance of vaccination in these neighborhoods during the second year of the project. The rapid vaccination phase of the intervention described here was carried out in Year 3, when the project aimed to deliver 1500 vaccines to persons within four specific communities of New York City during 10 working days. Years 4 and 5 are devoted to dissemination of project findings. 


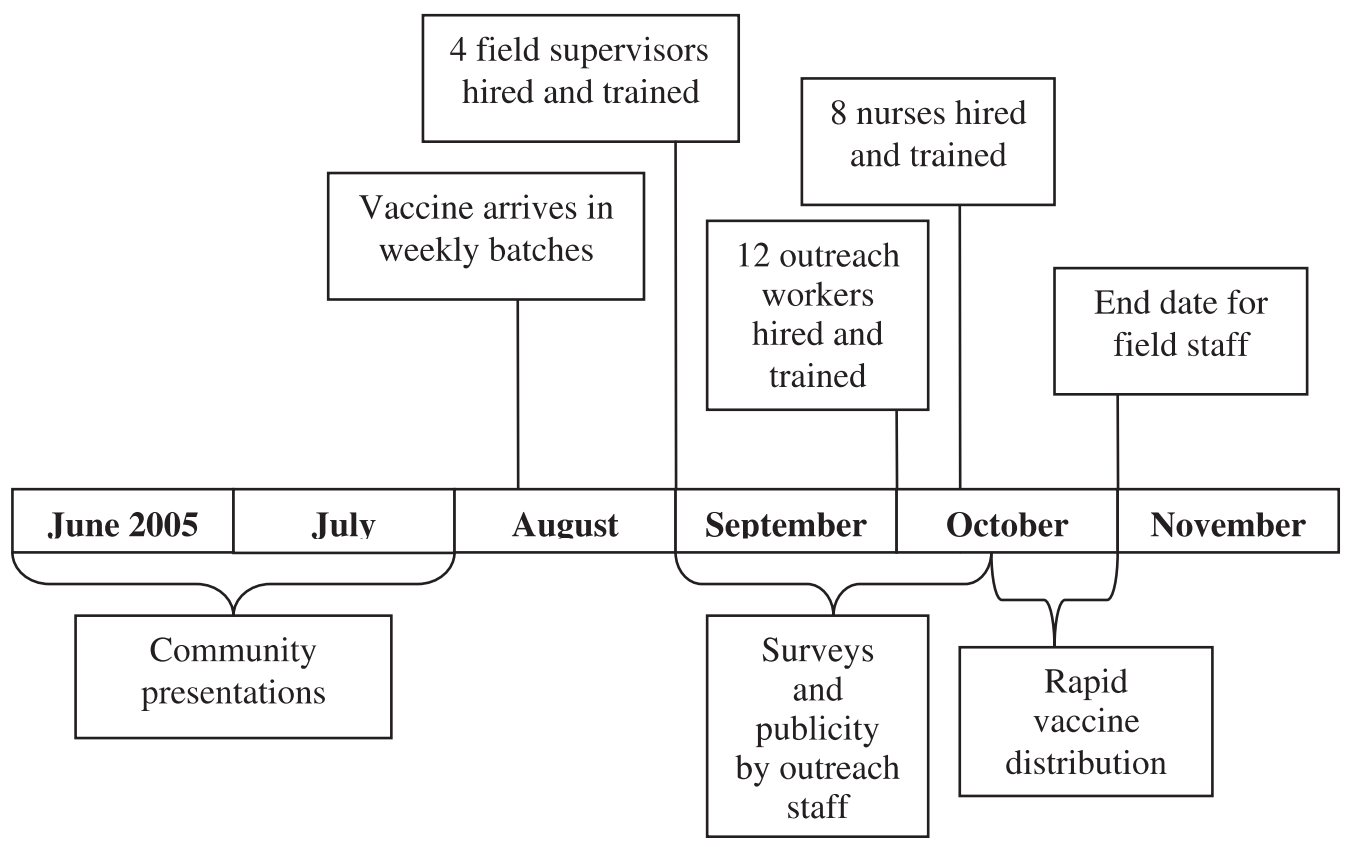

FIGURE 1 Project VIVA Rapid Vaccination Timeline.

\section{Rapid Vaccine Distribution}

Eight nurses were hired through a temporary nursing staffing agency to administer the vaccinations during the 10-day distribution phase (see Figure 1). Four bilingual (English/ Spanish) field supervisors were hired for a 2-month period to manage the outreach efforts, and 12 outreach workers were hired for 1 month to administer brief door-to-door and street-based surveys. All staff participated in a 2-day training covering study protocol, outreach role-plays, and the clinical aspects of the study (eligibility criteria, vaccine distribution, and handling adverse events). The project staff publicized the rapid vaccine effort by posting flyers that noted locations, dates, and times of vaccine distribution, and through presentations about the project at community meetings.

A team of two nurses, one field supervisor, and three outreach workers were assigned to each community (6-8 city blocks in size). Vaccines were offered door-to-door within apartment buildings and on the street. Members of the field staff were responsible for approaching potential participants, obtaining consent, and administering the study questionnaire. Vaccine eligibility was determined based on influenza vaccine guidelines; in addition, participants had to be $\geq 19$ years of age and not pregnant. Participants were vaccinated by the nurses while sitting on a portable seat brought to the field each day. 


\section{Adverse Events}

A detailed protocol was developed to handle adverse events, although none occurred. Staff members were assigned specific responsibilities (e.g., if warranted, nurses would administer epinephrine or Benadryl and maintain an airway; outreach workers would call 911 and wait for the ambulance). The medical director of the study, a physician, was on call at all times, and a licensed nurse managed all aspects of the program. Adverse events would have been reported to the NYAM Institutional Review Board and to the Vaccine Adverse Event Reporting System.

Flu vaccine was delivered to a total of 1,648 persons during the 10-day distribution phase, exceeding expectation. Almost half $(47 \%)$ of those vaccinated were members of a hard-to-reach population.

\section{LESSONS LEARNED}

This project demonstrated the feasibility of rapid vaccination of urban populations by community-based nurses. Overwhelmingly, reactions to the project were positive in the local communities and community members were eager to receive the vaccine. During the vaccination effort, the contact number provided on the publicity flyers yielded over 100 calls per week, indicative of the high level of community interest.

The project was originally designed to focus primarily on door-to-door vaccination; however, project staff quickly found door-to-door distribution to be relatively inefficient. Three visits were made to each apartment (on different days and at different times of the day) in an attempt to reach as many people as possible; however, vaccination rates were relatively low due to the number of people not at home during working hours and due to refusals. In contrast, field staff on the street, recognizable through their bright yellow Project VIVA jackets, were consistently approached by crowds of people interested in receiving the vaccine. Street-based distribution proved to be a very effective way to vaccinate large numbers of people in a brief time, and this method was given priority. Lines (up to 50 persons long) formed quickly for street-based distribution, although waiting time never exceeded $30 \mathrm{~min}$. The extent to which a particular mode of distribution was more effective for reaching specific vulnerable populations is still to be determined.

Although the aim of this project was to target hard-to-reach populations, project staff distributed vaccine to all interested and eligible persons in the targeted neighborhoods, irrespective of individual characteristics. Because the neighborhoods were low-income and much of the resident population lacked ready access to health care services, widespread distribution was warranted. In addition, the community-based methods-and the attention they garnered-contributed to improved access to hard-to-reach populations, 
and the resultant high percentage of vaccines being distributed to members of these groups.

Project staffing, training, and support, including the selection of nurses and outreach staff with complementary research skills and personal knowledge of the community, were key to project success. Community oversight of project development and implementation was essential, and greatly enhanced receptivity to the project. Project success was also facilitated by the community partnership's direction of the extensive outreach effort, both within the first 2 years of the study and immediately prior to the rapid vaccination phase, which allowed project staff and community members to become familiar with one another.

\section{IMPLICATIONS FOR OTHER RAPID VACCINATION PROGRAMS}

Developing strategies for vaccinating hard-to-reach populations in the event of an influenza pandemic is a priority in current federal preparedness plans. To best prepare the community for the eventuality of a pandemic, instituting annual mass flu vaccination campaigns in street-based nontraditional settings is recommended. Utilizing a community-based approach in project development and implementation will facilitate increased awareness and receptivity and maximize the success of the program. Outreach activities focused on promoting visibility and trust, including the obvious involvement of community nurses, should also be an integral part of this effort. Based on experience, this demonstration project, with nurses in a central community-based vaccine distribution role, has the potential to be generalized to other hard-to-reach populations in other areas, and utilized both for annual vaccination campaigns, and in the event of an influenza pandemic.

\section{REFERENCES}

Bryant, W. K., Ompad, D. C., Sisco, S., Blaney, S., Glidden, K., Phillips, E., et al. (2006). Determinants of influenza vaccination in hard-to-reach urban populations. Preventive Medicine, 43, 60-70.

Centers for Disease Control and Prevention. (2003). Racial/ethnic disparities in influenza and pneumococcal vaccination levels among persons aged $>$ or $=65$ years—United States, 1989-2001. MMWR Morbitity and Mortality Weekly Report, 52, 958-962.

Cinti, S. (2005). Pandemic influenza: Are we ready? Disaster Management Response, 3, 61-67.

D’Heilly, S. J., Lockman, J. L., \& Nichol, K. L. (2004). Adherence of mass vaccinators to timing guidelines for influenza vaccination. American Journal of Preventive Medicine, 26, 46-50.

Des Jarlais, D. C., Fisher, D. G., Newman, J. C., Trubatch, B. N., Yancovitz, M., Paone, D., et al. (2001). Providing hepatitis B vaccination to injection drug users: Referral to health clinics vs on-site vaccination at a syringe exchange program. American Journal of Public Health, 91, 1791-1792.

Egede, L. E., \& Zheng, D. (2003). Racial/ethnic differences in influenza vaccination coverage in high-risk adults. American Journal of Public Health, 93, 2074-2078. 
Findley, S. E., Irigoyen, M., See, D., Sanchez, M., Chen, S., Sternfels, P., et al. (2003). Community-provider partnerships to reduce immunization disparities: Field report from northern Manhattan. American Journal of Public Health, 93, 1041-1044.

Ho, G., \& Parker, J. (2006). Avian influenza: Risk, preparedness and the roles of public health nurses in Hong Kong. Nursing Inquiry, 13, 2-6.

Hoekstra, E. J., LeBaron, C. W., Megaloeconomou, Y., Guerrero, H., Byers, C., Johnson-Partlow, T., et al. (1998). Impact of a large-scale immunization initiative in the Special Supplemental Nutrition Program for Women, Infants, and Children (WIC). JAMA: Journal of the American Medical Association, 280, $1143-1147$.

Krieger, J. W., Castorina, J. S., Walls, M. L., Weaver, M. R., \& Ciske, S. (2000). Increasing influenza and pneumococcal immunization rates: A randomized controlled study of a senior center-based intervention. American Journal of Preventive Medicine, 18, 123-131.

Kuhles, D. J., \& Ackman, D. M. (2003). The federal smallpox vaccination program: Where do we go from here? Health Affairs (Millwood)(Suppl Web Exclusives), W3-10.

Kuo, I., Sherman, S. G., Thomas, D. L., \& Strathdee, S. A. (2004). Hepatitis B virus infection and vaccination among young injection and non-injection drug users: Missed opportunities to prevent infection. Drug and Alcohol Dependence, 73, 69-78.

Osterholm, M. T. (2001). How to vaccinate 30,000 people in three days: Realities of outbreak management. Public Health Reports, 116(Suppl 2), 74-78.

Poland, G. A., Grabenstein, J. D., \& Neff, J. M. (2005). The US smallpox vaccination program: A review of a large modern era smallpox vaccination implementation program. Vaccine, 23, 2078-2081.

Slobodkin, D., Kitlas, J., \& Zielske, P. (1998). Opportunities not missed-Systematic influenza and pneumococcal immunization in a public inner-city emergency department. Vaccine, 16, 1795-1802.

Stancliff, S., Salomon, N., Perlman, D. C., \& Russell, P. C. (2000). Provision of influenza and pneumococcal vaccines to injection drug users at a syringe exchange. Journal of Substance Abuse Treatment, 18, 263-265.

Weatherill, S. A., Buxton, J. A., \& Daly, P. C. (2004). Immunization programs in non-traditional settings. Canadian Journal of Public Health, 95, 133-137. 